\title{
Comparison of Conventional Tube and Gel-Based Agglutination Tests for AB System Blood Typing in Cat
}

\author{
Eva Spada*, Roberta Perego, Luciana Baggiani and Daniela Proverbio \\ Veterinary Transfusion Research Laboratory (REVLab), Department of Veterinary Medicine (DIMEVET), University of Milan, \\ Milan, Italy
}

Gel technology is widely used for blood typing in human medicine. It has a number of advantages over routine tube testing, including standardization, stability, smaller sample volume, ease of performance and analysis, and speed. The aim of this study was to evaluate feline blood typing using the gel column technique. TUBE agglutination typing was performed in 143 feline blood samples from blood donors and recipients, healthy and sick patients, and whole-blood units anticoagulated with ethylenediamine tetraacetic acid or citrate phosphate dextrose adenine. Plasma from type B cats was used as anti-A reagent, Triticum vulgaris lectin as anti-B reagent, and the control was saline solution. Agglutination in backtyping of types $B$ and $A B$ samples with type $A$ red blood cells (RBCs) was used to confirm whether the samples were type $B$ (presence of

OPEN ACCESS

Edited by:

Mehrdad Ameri,

GlaxoSmithKline, United States

Reviewed by:

Péter Vajdovich,

University of Veterinary Medicine

Budapest, Hungary

Labrini V. Athanasiou,

University of Thessaly, Greece

*Correspondence:

Eva Spada

eva.spada@unimi.it

Specialty section:

This article was submitted to Veterinary Experimental and

Diagnostic Pathology,

a section of the journa

Frontiers in Veterinary Science

Received: 26 February 2020

Accepted: 06 May 2020

Published: 23 June 2020

Citation:

Spada E, Perego R, Baggiani $L$ and Proverbio D (2020) Comparison of Conventional Tube and Gel-Based Agglutination Tests for AB System Blood Typing in Cat.

Front. Vet. Sci. 7:312. doi: 10.3389/fvets.2020.00312 alloantibodies) or type AB (absence of alloantibodies). Blood typing in a neutral gel column technique (GEL) using the same anti-A and anti-B reagents was performed on duplicate samples. Sensitivity, specificity, positive likelihood ratio, negative likelihood ratio, positive predictive value, negative predictive value, overall accuracy, and Cohen $\kappa$ coefficient (к) for GEL were calculated, with TUBE considered the gold standard technique. Of 143 samples typed with TUBE, 98 (68.5\%) were type A, 25 (17.5\%,) type B, and 20 (14.0\%) type AB. Backtyping confirmed the categorization of all types $B$ and $A B$ samples. Of these samples, gel testing produced 115 (80.4\%) concordant results; a mixed-field agglutination pattern (layers of RBCs at both the top and at the bottom of the gel in either the A or B gel column) was seen in 27 samples, and one type B sample was misidentified as type $A B$. If the mixed-field pattern was interpreted as a negative result, 141/143 (98.6\%) samples showed concordant results with an overall accuracy of the GEL of 100.0\% for type A, 98.9\% for type B, and 99.1\% for type AB. Strength of agreement was very good $(\kappa=0.97)$. When the same anti-A and anti-B reagents are used, GEL is a sensitive and specific method for blood typing feline samples. Until additional studies have been performed, mixed-field patterns obtained in GEL testing should be classified as negative results.

Keywords: cat, blood group, blood-typing method, agglutination, tube, gel, comparison

\section{INTRODUCTION}

The feline $\mathrm{AB}$ blood group system, characterized by three phenotypes: type $\mathrm{A}$, type $\mathrm{B}$, and type $\mathrm{AB}$, is the predominant blood group system in cats (1). In cats, naturally occurring alloantibody exists against the antigen they lack. Type A cats have low-titer antiB hemagglutinins and hemolysins. Type B cats have high-titer anti-A hemagglutinins and 
hemolysins. Type $\mathrm{AB}$ cats do not possess alloantibodies against either A or B antigens (2). These naturally occurring alloantibodies are responsible for transfusion reactions and neonatal isoerythrolysis in cats with incompatible blood types.

A knowledge of breed-related blood type and regional blood type epidemiology is useful when looking at the prevalence of different feline blood types. For example, the rare AB blood type is more common in some breeds, such as Ragdolls, in some countries (3). In addition, data on blood type epidemiology are useful when creating a blood bank by identification of breeds that may be preferentially recruited as blood donors. Maine Coon cats are large and quiet and have a high prevalence of A blood type (4). These data can be obtained by blood typing a large number of cats using a sensitive and specific test that can identify all three blood groups of the feline AB blood system.

Blood typing can be performed in-house or at a commercial laboratory. Currently, the most widely used in-house commercial blood typing kits include card agglutination tests and immunochromatographic tests (5-7). Commercial laboratories and blood banks usually employ the TUBE agglutination method.

The TUBE method is considered the gold standard for blood typing $(8,9)$. It requires the use of antigen-specific antisera and is performed by trained medical technologists. Despite being the gold standard in feline blood typing, the TUBE method has been difficult to standardize. In this test, the patient's red blood cells (RBCs) are incubated with antibodies against a specific blood type in a tube and then centrifuged and resuspended to assess for hemolysis or agglutination. The advantages of this method are that it can be completed in any practice, and there is no need for specialized equipment. The disadvantages are that it requires antigen-specific antisera, there is no universal protocol for completion and/or interpretation, it is time consuming (at least $1 \mathrm{~h}$ to complete), it requires advanced training, it produces subjective results, and it does not produce a stable reaction.

To overcome some of these disadvantages, gel technology has been widely used for blood typing in human medicine for many decades. The gel agglutination test (GEL) detects RBC antigen-antibody reactions using a chamber filled with polyacrylamide gel. The gel acts as a trap: free unagglutinated RBCs form pellets in the bottom of the tube (negative reaction); agglutinated $\mathrm{RBCs}$ remain at the top of the tube or are trapped in the gel (positive reaction). GEL testing has a number of important advantages over routine TUBE testing, particularly when testing large numbers of samples. These include standardization, stability, smaller sample volume, ease of performance and analysis, and rapidity. However, it does require specialized equipment.

Previously, a GEL test for feline blood typing was also available. It consisted of a dextran-acrylamide gel matrix impregnated with an antibody against the blood type of interest, and it was shown in previous studies to be the most accurate bedside test (99.4\% accuracy in cats) for feline AB blood typing $(5,8,9)$; unfortunately, this test is no longer available. Neutral human GEL columns with the addition of specific anti-RBC blood type antigen such as blood type 3, 4, 5, 7, Dal, and Kai reagents have been used in the last decade and proved useful in canine blood typing (10-12).
This gel agglutination technology could also be used for feline AB blood system blood typing. Therefore, the aim of this study was to evaluate the neutral human gel column technique in $A B$ blood system feline blood typing, comparing the results with the TUBE gold standard technique.

\section{MATERIALS AND METHODS \\ Blood Samples}

Ethylenediamine tetraacetic acid (EDTA)-anticoagulated surplus blood samples used for blood typing cats for clinical reasons (blood donor, blood recipient, blood typing before mating, or as part of preoperative evaluation) at the Veterinary Transfusion Research Laboratory (REVLab) of the University of Milan were used in this study. The blood was stored at $4^{\circ} \mathrm{C}$ and typed within 7 days of collection.

During consultations and prior to blood donation or transfusion, owners provided written consent for blood collection, use of blood samples, and use of data for scientific purposes. Ethical review and approval were not required for the animal study because only surplus EDTA blood samples drawn for routine diagnostic work were used.

For each blood sample tested, the cat's age, breed, and sex were recorded. When available, the cat's health status or underlying disease was also recorded. Packed Cell Volume (PCV) and autoagglutination were recorded.

Duplicate samples were blood typed using the same anti$A$ and anti-B reagents in both the TUBE and neutral GEL column technique.

\section{Blood Typing by Tube Technique}

TUBE was performed with slight modifications as previously described $(2,13)$.

To prepare an RBC suspension for the TUBE method, $1 \mathrm{~mL}$ of anticoagulated blood was centrifuged for $10 \mathrm{~min}[1,600 \mathrm{~g}$ at room temperature $\left.\left(\sim 20^{\circ} \mathrm{C}\right)\right]$. Plasma was collected into a separate tube and stored for possible backtyping or other analysis. The RBC pellet was resuspended in $5 \mathrm{~mL}$ of isotonic $0.9 \%$ saline $\mathrm{NaCl}$ solution. The suspension was then recentrifuged and resuspended three times and finally reconstituted to a $5 \%$ RBC suspension.

Polyclonal antibodies contained in type B cat plasma (obtained from a type B blood donor, collected with CPD anticoagulant at a ratio of $1: 7$, stored at $-20^{\circ} \mathrm{C}$ ) were used as primary reagents for the detection of type A red cell antigens. Triticum vulgaris lectin $[8 \mu \mathrm{g} / \mathrm{mL}$, made by mixing $2 \mathrm{mg}$ of stock solution with $250 \mathrm{~mL}$ phosphate-buffered saline (PBS), stored, aliquoted in $10 \mathrm{ml}$ tube at $-20^{\circ} \mathrm{C}$ ] (Sigma-Aldrich Corporation, MERK KGaA Darmstadt, Germany) was used for the detection of type B RBC antigens as this lectin binds to the NeuAc terminal of the type B ganglioside (13). A $0.9 \%$ saline solution was used as a negative control and to test for autoagglutination.

In three glass tubes, $50 \mu \mathrm{L}$ of $5 \% \mathrm{RBC}$ suspension was mixed with $100 \mu \mathrm{L}$ of type B plasma (anti-A reagent), $100 \mu \mathrm{L}$ of T. vulgaris lectin solution (anti-B reagent), or $100 \mu \mathrm{L}$ of saline solution (control reagent), respectively. These mixtures were incubated at room temperature for $15 \mathrm{~min}$ before centrifugation 


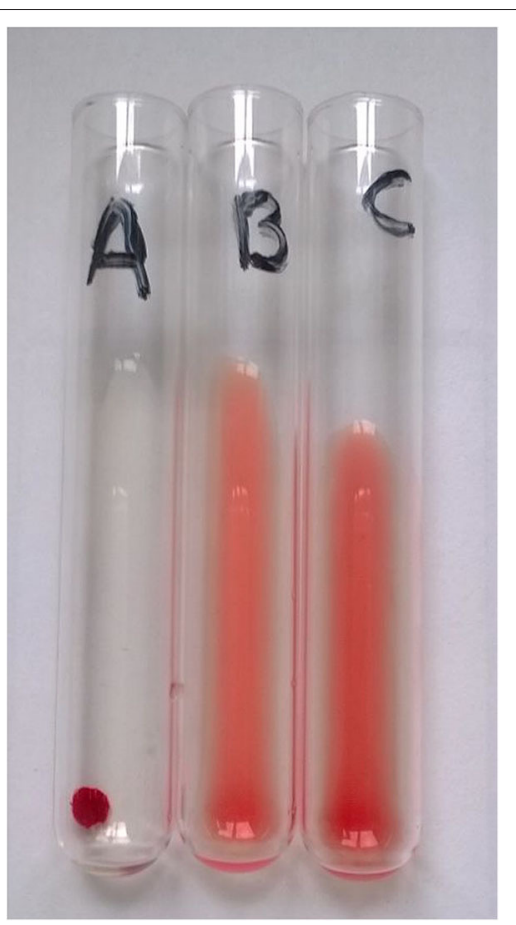

FIGURE 1 | Example of type A blood sample typed using the tube agglutination technique (TUBE). Total clumping of red cells in "A" tube and absence of agglutination in "B" and "C" (control) tubes identify this sample as type A with maximum strength of agglutination (4+).

for $15 \mathrm{~s}$ at $1,000 \mathrm{~g}$. Tubes were then gently agitated, and agglutination was scored from 0 (no agglutination, negative reaction) to $4+$ (single pellet-like agglutination, maximum grade of positive reaction, Figure $\mathbf{1}$ ).

\section{Blood Typing by Gel Method}

Duplicate samples were blood typed with a neutral gel column technique using the same anti-A and anti-B reagents as in the TUBE technique. The GEL test consists of a card with six microtubes for determining the blood types of two samples. Each microtube contains a neutral gel matrix (ID-Card $\mathrm{NaCl}$ enzyme test and cold agglutinins; DiaMed $\mathrm{GmbH}$, Cressier FR, Switzerland).

Red blood cells were first separated from plasma by centrifugation at $1,600 \mathrm{~g}$ for $10 \mathrm{~min}$, and a $0.8 \% \mathrm{RBC}$ suspension prepared, by suspending $10 \mu \mathrm{L}$ of the RBC pellet in $1 \mathrm{~mL}$ of low ionic strength solution (ID-Diluent 2; DiaMed) provided by the same manufacturer.

A $50 \mu \mathrm{L}$ RBC suspension from each sample was loaded on the top of three gel columns (reaction chamber) and mixed with 25 $\mu \mathrm{L}$ of type B plasma, $25 \mu \mathrm{L}$ of $T$. vulgaris lectin stock solution, and $25 \mu \mathrm{L}$ of PBS in the A, B, and negative control (ctl) columns, respectively. Control columns that contained gel with PBS only were included to detect potential false-positive reactions due to autoagglutination.

Following incubation at room temperature $\left(\sim 20^{\circ} \mathrm{C}\right)$ for $15 \mathrm{~min}$, the gel columns were centrifuged in a special gel column

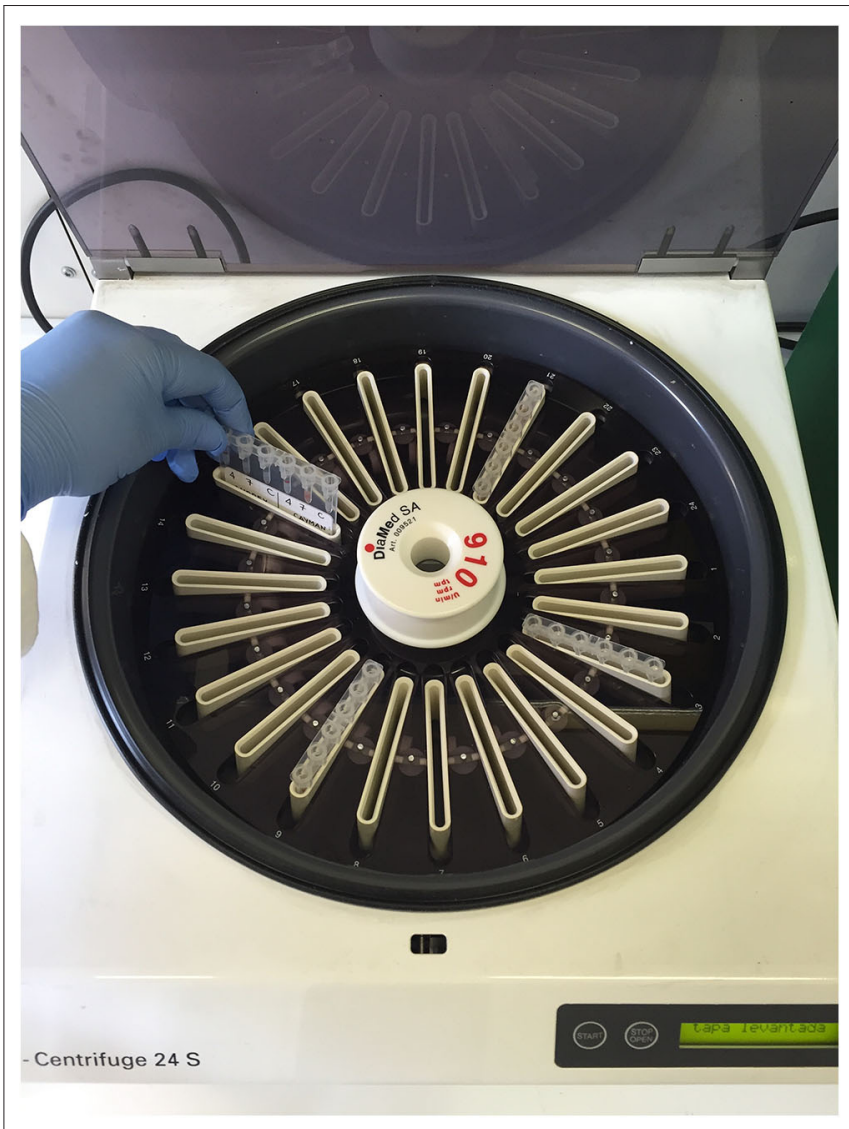

FIGURE 2 | Special gel column card centrifuge in which the gel column cards were centrifuged at $80 \mathrm{~g}$ for $10 \mathrm{~min}$.

card centrifuge (ID-Centrifuge 24 S; DiaMed; Figure 2) at $80 \mathrm{~g}$ for $10 \mathrm{~min}$. The results were viewed and graded from negative to $4+$ depending on the location of the majority of RBCs within the gel column, as follows: $4+$ : all RBCs were agglutinated and formed a red line on the surface of the gel; 3+: most RBCs were agglutinated and formed a red line on the surface of the gel; 2+: all RBC agglutinates were dispersed in the gel; $1+$ : few RBC agglutinates were dispersed in the gel, and most of the RBCs were at the bottom of the tube; 0 (negative): all $\mathrm{RBCs}$ were at the bottom of the tube (not agglutinated). If the A- or B-labeled column had a positive reaction, the cat had type A or type B blood, respectively; if both columns were positive, the cat had type $\mathrm{AB}$ blood (Figures 3, 4). Red blood cell retention of $\geq 1+$ was considered a positive test result. The test was considered invalid if the ctl-labeled column was not consistently negative.

\section{Back Typing}

Backtyping technique or antibody screening is a modified major crossmatch using RBCs of a known blood group, thus screening for recipient antibodies against that group.

To confirm types $\mathrm{B}$ and $\mathrm{AB}$ samples, all samples were tested for presence (type $B$ ) or absence (type $A B$ ) of anti-B isoagglutinins 


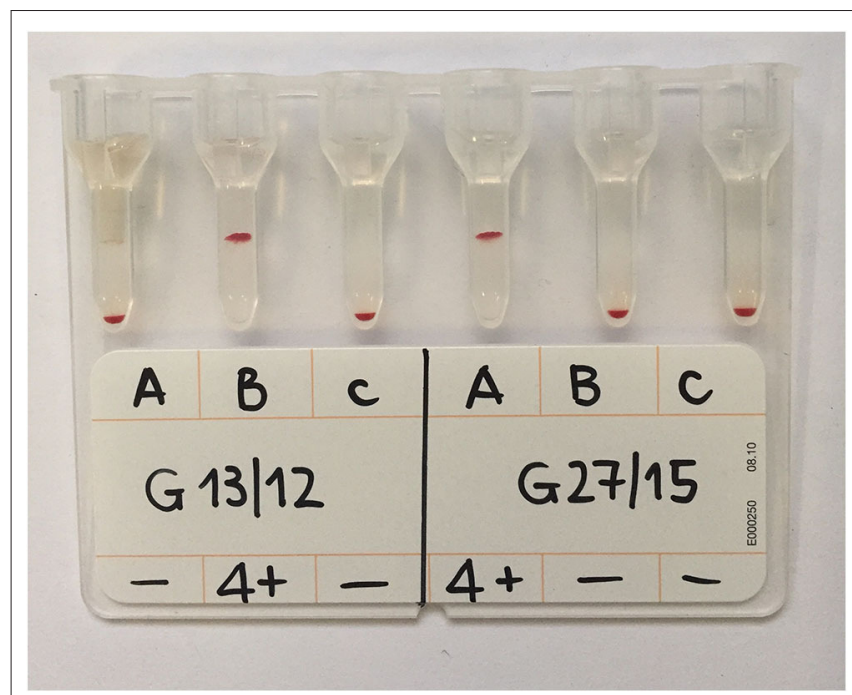

FIGURE 3 | GEL column card with two analyzed samples. The sample labeled G13/12 was type B blood as the agglutination occurred only in the B column; sample G27/15 was type A as agglutination occurred only in the A column. Both reactions were graded $4+$ as all the RBCs were agglutinated and formed a layer at the top of the gel column. Control columns (c) containing PBS only are included and show absence of autoagglutination.

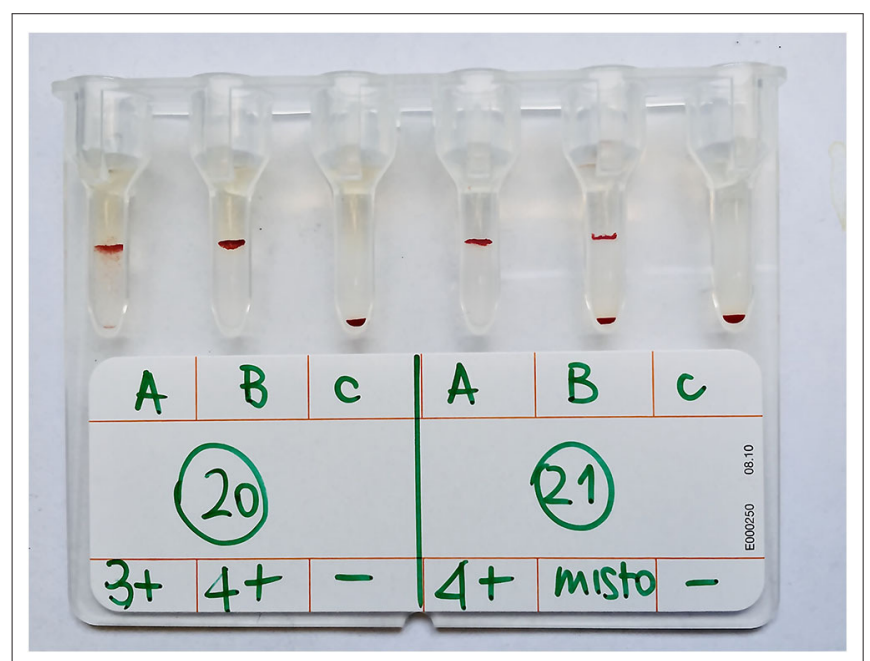

FIGURE 4 | Example of samples with gel agglutination mixed-field pattern results. Sample $n .21$ showed a mixed-field pattern (identified as "misto" in the Figure) in B column and was classified as A blood type based on comparison with agglutination on tube technique, the gold standard technique. Sample $n$. 20 showed agglutination in both $A$ and $B$ columns and was $A B$ blood type, as confirmed by agglutination on tube technique. Control columns (c) containing only PBS are included and show negative test (absence of autoagglutination).

using a backtyping technique as previously described $(3,8,9)$. Briefly, in a glass tube, $25 \mu \mathrm{L}$ of the type A RBC suspension $(5 \%$ in $0.9 \% \mathrm{NaCl}$ ) was added to $50 \mu \mathrm{L}$ of type $\mathrm{B}$ or $\mathrm{AB}$ plasma. The solution was gently mixed, incubated for $15 \mathrm{~min}$ at room temperature $\left(\sim 20^{\circ} \mathrm{C}\right)$, and then centrifuged for $15 \mathrm{~s}$ at $1,000 \mathrm{~g}$. Agglutination was evaluated by gently agitating the tube to resuspend the non-agglutinating RBCs in the cell button. Plasma from type $\mathrm{B}$-positive cats showed a strong agglutination reaction with type A-positive RBCs, whereas plasma from AB-positive cats did not agglutinate with type A.

\section{Additional Analysis}

To better understand the reasons for mixed-field pattern results in GEL, stored plasma samples $\left(-20^{\circ} \mathrm{C}\right)$ of most blood samples showed this mixed-field pattern and available plasma from all other samples were retrospectively tested for feline leukemia virus infection (FeLV) and for feline immunodeficiency virus infection (FIV). Presence of antibodies to FIV target antigens p24 and gp40 and of FeLV p27 antigen was simultaneously checked on plasma using a commercial rapid enzyme-linked immunosorbent assay kit (SNAP ${ }^{\circledR}$ Combo Plus FeLV Ag/FIv Ab; IDEXX Laboratories Italia S.r.l., Milan, Italy).

\section{Statistical Analysis}

As suggested by the National Committee for Clinical Laboratory Standards guidelines, a minimum of 40 samples from different patients has been tested by the two methods (14). These samples were selected to cover the entire working range of the method, that is, all three blood types, type A, B, and AB, and should represent the distribution of blood types expected in routine application of the method. However, more blood samples obtained from type $\mathrm{AB}$ or $\mathrm{B}$ cats were included in the study to assist recognition of any potential typing problems.

Sensitivity (Se), specificity (Sp), positive likelihood ratio (PLR), negative likelihood ratio (NLR), positive predictive value (PPV), negative predictive value (NPV), and overall accuracy for GEL were calculated, with TUBE results considered the gold standard. For this method's comparison study, samples were preferentially obtained from type $\mathrm{AB}$ or $\mathrm{B}$ cats to assist in recognizing any unique potential typing problem. Therefore, as the sample sizes for each blood type used in this study did not reflect the real prevalence of $\mathrm{AB}$ blood types in the tested feline population, to evaluate use as a diagnostic test, the real data prevalence from a previous study performed on 140 cats living in the same area and tested by the same authors of this study was used (5), that is, a prevalence of $90.7 \%$ for type A, $7.1 \%$ for type $B$, and $2.1 \%$ for type $A B$.

An interrater agreement, unweighted $\kappa$ statistic ( $\kappa)$ was calculated with 95\% confidence interval (95\% CI) to evaluate agreement between the two testing methods in detecting blood type (15). The level of agreement based on value of $\kappa$ was scored according to the following guidelines (16):

$$
\begin{aligned}
& <0.20 \text { : Poor } \\
& 0.21-0.40 \text { : Fair } \\
& 0.41-0.60: \text { Moderate } \\
& 0.61-0.80: \text { Good } \\
& 0.81-1.00: \text { Very good }
\end{aligned}
$$

Relationship between results of FIV-FeLV test and samples showing mixed-field pattern in GEL technique was tested with Fisher exact test with significance set at $P<0.05$. 
All statistical analyses were performed using a statistical software package (MedCalc Software, version 19.1.3; Mariakerke, Belgium).

To test the intra-assay precision, the GEL was performed six times on the same day on a single sample of type A, B, and AB. All tests were run in the same laboratory and interpreted by the same technician blinded to previous results.

Five samples of each of type $A, B$, and $A B$ were tested in a blinded fashion by three different operators (a technician, a veterinarian, and a student) to establish the interassay accuracy of GEL test results.

One sample of each blood type stored at room temperature and at $4 \pm 2{ }^{\circ} \mathrm{C}$ was analyzed with GEL at T0, after 24 and $48 \mathrm{~h}$, and after 1,2,3, and 4 weeks of storage.

At D0 (the day of collection of the unit) and on D28 (day of the expiration of the unit), GEL was performed on five samples of type A citrate phosphate dextrose adenine (CPDA) anticoagulated blood drawn from both fresh and stored wholeblood units.

\section{RESULTS}

One hundred forty-three blood samples were tested in this study and were from 127 European domestic shorthair, 7 Ragdolls, 3 Persians, 2 Maine Coons, 1 Norwegian forest cat, 1 British shorthair cat, 1 Sphynx, and 1 Siamese. Fifty-seven samples were from female cats and 86 from male, aged between 1 month and 18 years [mean, 4.3 (SD 4.1) years]. Ten cats were blood recipients, 13 were blood donors, 15 were blood typed for breeding purposes, 18 as a preoperative check, and 87 cats were blood typed as part of an epidemiological study (data/results not shown).

Mean PCV was 30.3\% (SD, 9.9\%; min-max, 9.0-46.7\%); three samples showed autoagglutination, and 15 samples were from anemic cats $[\mathrm{PCV}<24 \%$; mean, $17.2 \%$ (SD, 5.4\%); min$\max , 9.0-23.9 \%]$.

Of 143 samples typed with TUBE, 98 (68.5\%) were type A, $25(17.5 \%$,) type B, and $20(14.0 \%)$ type AB. Most agglutination reactions were strongly positive $(4+$ or $3+$ ). All types $B$ and $A B$ samples were confirmed by results of backtyping, with strong agglutination reaction and no agglutination in types $B$ and $A B$ plasma samples, respectively, crossmatched against type A RBCs.

With GEL, 115/143 samples (80.4\%) gave concordant results with TUBE; a mixed-field agglutination pattern (presence of a layer of RBCs at the top and bottom of A or B gel column, Figure 4) was seen in 27 samples (18.9\%), and one type B sample was misidentified as type $A B$ (Table 1). As in TUBE, most agglutination reactions were strongly positive $(4+$ or $3+$ ). Results of Se, Sp, PLR, NLR, PPV, NPV, and accuracy are reported in Table 2. Strength of agreement of two methods in blood typing when mixed-field pattern results were considered positive was good $(\kappa=0.67$; standard error, $0.05 ; 95 \%$ CI, 0.56-0.77).

Twenty of 27 samples with GEL mixed-field pattern were tested for FIV and FeLV infection, and four samples tested FIV seropositive. Thirty-six of 116 samples with normal GEL pattern
TABLE 1 | Feline blood type results of 143 EDTA blood samples tested with agglutination on TUBE (gold standard) and GEL techniques where gel mixed-field pattern results were considered positive results.

\begin{tabular}{lcccc}
\hline Gel agglutination & \multicolumn{4}{c}{ Tube agglutination (gold standard) } \\
\cline { 2 - 5 } & A & B & AB & Total (\%) \\
\hline A & 76 & 0 & 0 & $76(53.1)$ \\
B & 0 & 20 & 0 & $20(14.0)$ \\
AB & $22^{*}$ & $5^{\star *}$ & $20^{\star \star \star}$ & $47(32.9)$ \\
Total (\%) & $98(68.5)$ & $25(17.5)$ & $20(14.0)$ & $143(100)$
\end{tabular}

${ }^{*}$ In all these samples, a mixed-field pattern in B GEL column was identified. ${ }^{* *}$ In four of these samples and in ${ }^{* \star *}$ one of these samples, a mixed-field pattern in A GEL column was identified and interpreted as a positive result, that is, test positive for agglutination.

were tested; one was FIV seropositive, and one FeLV seropositive. Relationship between retrovirus seropositivity and presence of GEL mixed-field pattern was not significant $(P=0.17)$.

If GEL mixed-field pattern results were interpreted as negative results (as gold standard method showed no agglutination), 141/143 (98.6\%) samples showed concordant results (Table 3) with Se, Sp, PLR, NLR, PPV, NPV, and accuracy as reported in Table 4. One TUBE type B sample was identified as type AB by GEL and one TUBE type AB as B by GEL. Strength of agreement of two methods in blood typing was very good $(\kappa=0.97$; standard error, 0.02; 95\% CI:0.93-1.00).

All anemic and autoagglutinated samples were correctly typed by GEL.

Single samples of types A, B, and AB blood typed multiple times with GEL were always correctly blood typed. The GEL method showed $100 \%$ intra-assay accuracy.

All five samples of types A, B, and AB were correctly identified giving an excellent interassay accuracy. Interassay accuracy was not affected by using three different operators.

Blood samples stored at room temperature and at $4 \pm 2{ }^{\circ} \mathrm{C}$ for up to 1 month were correctly typed by GEL-as were all samples from whole-blood units anticoagulated with CPDA1 on D0 and on D28 of storage.

\section{DISCUSSION}

Feline transfusion medicine has grown rapidly in the last decades, and today, many sick cats receive blood transfusion, and many healthy cats donate blood. Even a small quantity of incompatible blood in the first blood transfusion could cause an acute hemolytic transfusion reaction that could be fatal for type B cats receiving type A blood (17). In all other incompatible blood transfusions, the failure to type blood correctly could result in a less effective transfusion, wasting a very precious and limited resource (18). Therefore, it is imperative to have sensitive and specific blood-typing methods in both emergency situations, in which fast and precise immunomigration tests are needed, and in routine and epidemiological settings, where high sensitivity and specificity can be combined with easy, rapid and economic test characteristics. 
TABLE 2 | Diagnostic performances of a GEL technique compared to a TUBE technique in feline AB system blood typing of 143 feline whole-blood samples anticoagulated with EDTA, considering GEL mixed-field pattern results as positive results.

\begin{tabular}{|c|c|c|c|c|c|c|}
\hline \multirow[t]{3}{*}{ Statistics } & \multicolumn{6}{|c|}{$\begin{array}{l}\text { Blood type with GEL (considering mixed-field pattern as positive results) } \\
\text { compared to TUBE technique }\end{array}$} \\
\hline & \multicolumn{2}{|c|}{ A } & \multicolumn{2}{|c|}{ B } & \multicolumn{2}{|c|}{$A B$} \\
\hline & Value (\%) & $95 \% \mathrm{Cl}$ & Value (\%) & $95 \% \mathrm{Cl}$ & Value (\%) & $96 \% \mathrm{Cl}$ \\
\hline Sensitivity (Se) & 77.55 & $68.01-85.36$ & 80.00 & 59.30-93.17 & 100.00 & 83.16-100.00 \\
\hline Specificity (Sp) & 100.00 & $92.13-100.00$ & 100.00 & $96.92-100.00$ & 78.05 & 69.69-85.01 \\
\hline Positive likelihood ratio (PLR) & - & - & - & - & 4.56 & $3.26-6.36$ \\
\hline Negative likelihood ratio (NLR) & 0.22 & $0.16-0.32$ & 0.20 & $0.09-0.44$ & 0.00 & - \\
\hline Positive predictive value (PPV) & 100.00 & - & 100.00 & - & 9.90 & $6.54-12.00$ \\
\hline Negative predictive & 31.35 & $24.02-39.76$ & 98.49 & $96.76-99.31$ & 100.00 & - \\
\hline Accuracy & 79.64 & 72.10-85.91 & 98.58 & $95.01-99.82$ & 78.51 & $70.87-84.93$ \\
\hline
\end{tabular}

TABLE 3 | Feline blood type results of 143 EDTA blood samples with agglutination on tube (gold standard) and with gel techniques where GEL mixed-field pattern results considered negative results.

\begin{tabular}{lcccc}
\hline Gel agglutination & \multicolumn{4}{c}{ Tube agglutination (gold standard) } \\
\cline { 2 - 5 } & A & B & AB & Total (\%) \\
\hline A & $98^{\star}$ & 0 & 0 & $98(68.5)$ \\
B & 0 & $24^{\star \star}$ & $1^{* \star \star}$ & $25(17.5)$ \\
AB & 0 & 1 & 19 & $20(14.0)$ \\
\hline Total & $98(68.5)$ & $25(17.5)$ & $20(14.0)$ & $143(100)$
\end{tabular}

*Twenty-one samples showed a mixed pattern in the B GEL column and were interpreted as negative results/absence of agglutination. ${ }^{* *}$ Four of these samples showed a mixed pattern in the A GEL column and were interpreted as negative results, that is, absence of agglutination, ${ }^{\star \star *}$ This sample showed a mixed pattern in the A GEL column and was interpreted as negative.

Accurate blood typing is often difficult to achieve in sick patients. Many feline patients received Whole Blood (WB) transfusion for anemia. Anemia may be severe in these cases, and sometimes these patients show persistent autoagglutination of blood samples. Severe anemia and autoagglutination can pose problems in blood typing, especially when using agglutination on cards or tests based on RBCs migration $(7,9,19)$. The TUBE, technique overcomes problems related to severe anemia, as centrifugation concentrates RBCs in the sample, and relatively few RBCs are needed to run these tests. All anemic samples in our study were correctly typed, even when PCV was as low as $9 \%$. Autoagglutination can be detected in the TUBE and GEL methods if a control reaction with saline or PBS is run to reveal false-positive results due to autoagglutinated samples. In addition, the TUBE method is useful in autoagglutination as the few non-agglutinating RBCs in these samples are sufficient to detect the blood type, as confirmed by our study when three autoagglutinated samples were correctly typed by GEL.

The most important problem with GEL in AB feline group blood typing highlighted by this study was the occasionally mixed-field pattern result, which makes interpretation difficult without a reference to another method of typing. Mixed-field pattern results have been reported by other researchers in a limited number of veterinary publications. In a sample from a cat with FeLV-related anemia, the GEL test reaction showed a split population of RBCs in the anti-B column, making the results inconclusive (9). Our results suggest that retrovirus infections, that is, FIV or FeLV infections, do not result in GEL mixedfield patterns.

In a few cases, Euler et al. (12) observed an unexplained split reaction in Kai canine blood typing, with the majority of cells located on top of the gel despite a few RBCs being pelleted. These cases were classified as $4+$ results. These dogs had not been previously transfused, nor had any illness (12). In human medicine, GEL mixed-field patterns arise from (I) mixed RBCs populations from recent blood transfusions (presence of circulating donor red cells in recipient), transplanted bone marrow or peripheral blood stem cells of a different $\mathrm{ABO}$ type, exchange transfusions, fetal-maternal hemorrhage, blood group chimerism in fraternal twins, mosaicism arising from dispermy (20, 21); (II) false-positive reactions when incompletely clotted serum is used (21); (III) various diseases that alter $R B C$ antigens and result in progressively weaker reactions or additional acquired pseudoantigens (e.g., leukemia, Hodgkin disease, thalassemia) (22); (IV) some $A B O$ subgroups (21). Considering that $18.9 \%$ of the samples analyzed in this study with GEL showed a mixed-field pattern, it is improbable that all these patients were affected by chimerism or mosaicism or had received recent blood transfusion that transiently changed their blood type. As the type B plasma used as anti-type A reagent was the same in both methods (GEL and TUBE) and for all samples, this should not be the cause of mixedfield pattern. As health information related to blood samples analyzed in this study was collected retrospectively, we have limited information on some patients, and apart from the possible presence of diseases such as FeLV or FIV infection, we have limited data on presence of neoplasia, feline immunemediated hemolytic anemia, or severe inflammation, which could alter RBC antigens $(9,23)$. Therefore, the possibility that some disease could be the cause of this mixed-field pattern cannot be excluded. It is also possible that subtle or microscopic 
TABLE 4 | Diagnostic performances of a GEL technique compared to a TUBE technique in feline AB system blood typing of 143 feline whole-blood samples anticoagulated with EDTA, where GEL mixed pattern results were considered as negative results.

Statistics Blood type with GEL (considering mixed-field pattern as negative results) compared to TUBE technique

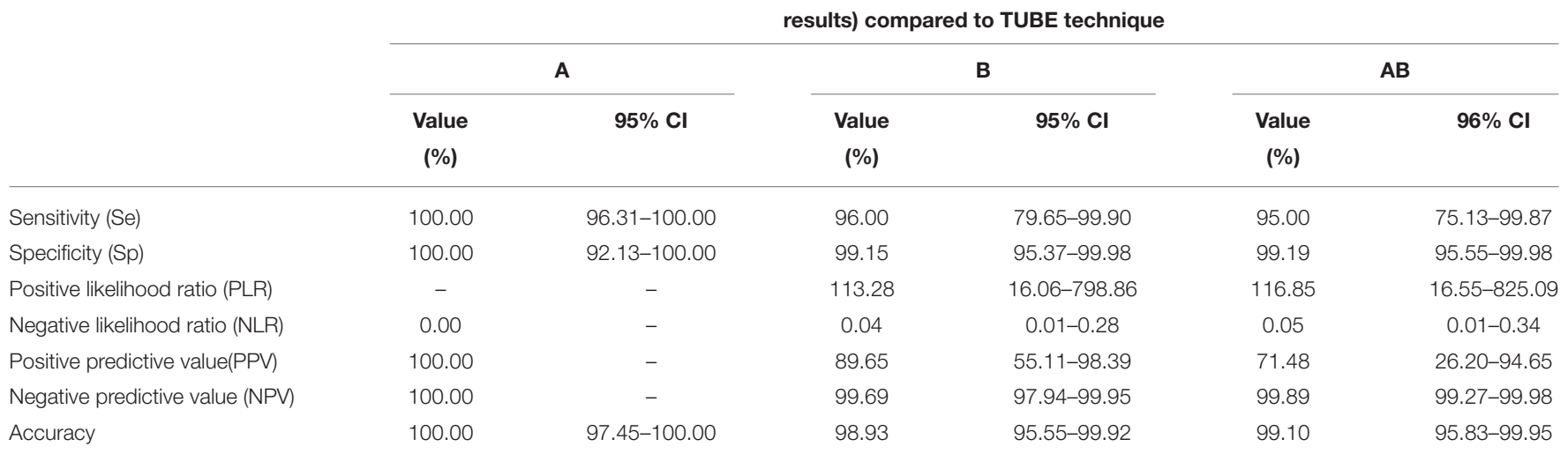

autoagglutination that was not detected by TUBE resulted in the GEL mixed-field pattern due to a reaction with $\mathrm{RBC}$ antigens from either a blood type outside the $\mathrm{AB}$ blood type system such as Mik (24) or, as previously demonstrated, the presence of natural occurring alloantibodies outside the $\mathrm{AB}$ blood group system $(25,26)$. To prevent nonspecific agglutination reactions causing blood type misclassification, monoclonal antibodies to type A RBC would be the ideal reagent for the future use in GEL methods.

The characteristic of the samples could be responsible for the mixed-field pattern. Mixed-field reactions can occur when incompletely clotted serum is used in the gel test in human medicine. Fibrin strands in serum may trap unagglutinated RBCs, forming a thin line at the top of the gel, whereas other unagglutinated cells pass through the gel during centrifugation and fall to the bottom of the microtube (21). Rouleaux caused by serum or plasma with abnormally high concentrations of protein may produce hazy reactions or false-positive results (21). Presence of rouleaux is a frequent hematological feature in diseased (but also in many healthy) cats (27). Future studies should therefore explore reasons for GEL mixed-field patterns looking for the presence of hyperproteinemia or presence of rouleaux and trying to repeat the test in samples with GEL mixed-field pattern after washing of RBCs to disperse rouleaux as performed in the TUBE technique.

However, if the mixed-field patterns were considered as negative results, there were only two discordant results between TUBE and GEL. One was a TUBE type AB cat typed as B by GEL. This sample come from a young female cat with recent Giardia infestation that was blood typed before surgery for spaying and was FIV and FeLV negative. The second discordant result was a type $B$ sample misclassified as type $A B$ by GEL from a healthy young cat blood typed before routine spaying. Unfortunately, we have no additional information on this cat, and it was not possible to test for FIV and FeLV infections as there was insufficient sample. Misclassifying a type B cat as type $\mathrm{AB}$ could have serious clinical consequences if testing a blood recipient, as a type $\mathrm{AB}$ cat could receive type $\mathrm{A}$ blood with no (or limited) clinical sequela, but in a type B cat, a small amount of type A blood could result in a fatal hemolytic reaction. However, following good practices, crossmatching should be done before every blood transfusion, which would reveal a blood incompatibility between types A and B blood, and other blood types such as Mik (24), to avoid hemolytic transfusion reactions.

Although the TUBE method is considered the gold standard technique in feline blood typing $(8,9)$, the GEL test provides major advantages in $\mathrm{AB}$ blood typing compared with traditional tube technology $(21,28)$. The most important advantages in veterinary medicine appear to be (I) rapidity as, with GEL, the blood type was established in half the time of the TUBE method (mostly because GEL does not require the washing of RBCs, which reduced the overall time to obtain blood type): this advantage is particularly important in emergency situation or in epidemiological studies when many samples are analyzed as a batch; (II) stable, well-defined endpoints of the agglutination reaction that can be reviewed for up to 3 days: the result can be photographed and archived, viewed by multiple technicians at different times, whereas, with TUBE, the endpoint reaction is stable for only a few hours; (III) more objective and consistent results as the endpoint agglutination results are more clear and simple to read, grade, and interpret; (IV) standardization, because there is no tube shaking to resuspend the RBC button, the variability associated with the physical resuspension of RBC buttons after centrifugation and the subsequent interpretation of hemagglutination reactions are reduced, permitting more reproducible test results; (V) reduced volume of sample and reagents as only $10 \mu \mathrm{L}$ of the $\mathrm{RBC}$ pellet is required: this is particularly useful when working with small animals such as cats for which the blood sample volume is often limited. In addition, type B plasma sample used as anti-A reagent can be saved and used at a quarter of the volume used in TUBE, preserving an important biological reagent; (VI) fewer operator interventions, thus reducing technical error. For all these reasons, some veterinary laboratories, particularly those working with large number of samples or needing rapid results for emergency 
situations, might prefer GEL over TUBE in blood typing feline samples.

This study did not evaluate the agreement between methods for agglutination strength reactions. In tests based on agglutination using T. vulgaris lectin as anti-B reagent and type $B$ plasma as anti-A reagent, the reactions are usually strong, $3+$ or $4+$, as seen in the samples analyzed in this study. As the objective of the study was to compare GEL to TUBE technique, most samples had incomplete clinical information. Therefore, we cannot rule out the possibility that some animals may have had infections or diseases other than retrovirus infections, such as neoplasia, feline immune-mediated hemolytic anemia, or severe inflammation, which could alter RBC antigens.

In conclusion, if the same anti-A and anti- $\mathrm{B}$ reagents are used as in TUBE testing, the GEL column technique is a sensitive and specific method for typing feline blood samples, with a number of advantages over the TUBE technique. Until additional studies improve our understanding of the significance of mixedfield pattern in GEL technology, a mixed-field pattern should be considered as a negative result in veterinary transfusion medicine. As TUBE is the only method that has $100 \%$ sensitivity and specificity in detecting $\mathrm{B}$ and $\mathrm{AB}$ blood types, crossmatching before blood transfusion remains key to prevent fatal incompatible transfusion reactions.

\section{DATA AVAILABILITY STATEMENT}

The datasets generated for this study are available on request to the corresponding author.

\section{REFERENCES}

1. Auer L, Bell K. The AB blood group system of cats. Anim Blood Groups Biochem Genet. (1981) 12:287-97. doi: 10.1111/j.1365-2052.1981.tb01561.x

2. Bücheler J, Giger U. Alloantibodies against A and B blood types in cats. Vet Immunol Immunopathol. (1993) 38:283-95. doi: 10.1016/ 0165-2427(93)90088-L

3. Proverbio D, Spada E, Perego R, Della Pepa A, Bagnagatti De Giorgi G, Baggiani L. Assessment of blood types of Ragdoll cats for transfusion purposes. Vet Clin Pathol. (2013) 42:157-62. doi: 10.1111/vcp.12048

4. Spada E, Antognoni MT, Proverbio D, Ferro E, Mangili V, Miglio A. Haematological and biochemical reference intervals in adult Maine Coon cat blood donors. J Feline Med Surg. (2015) 17:1020-7. doi: 10.1177/1098612X14567549

5. Proverbio D, Spada E, Baggiani L, Perego R, Milici A, Ferro E. Comparison of gel column agglutination with monoclonal antibodies and card agglutination methods for assessing the feline $\mathrm{AB}$ group system and a frequency study of feline blood types in northern Italy. Vet Clin Pathol. (2011) 40:329. doi: 10.1111/j.1939-165X.2011.00286.x

6. Spada E, Proverbio D, Baggiani L, Bagnagatti De Giorgi G, Perego R, Ferro E. Evaluation of an immunochromatographic test for feline AB system blood typing. J Vet Emerg Crit Care. (2016) 26:137-41. doi: 10.1111/vec.12360

7. Hourani L, Weingart C, Kohn B. Evaluation of a novel feline AB blood typing device. J Feline Med Surg. (2014) 16:826-31. doi: 10.1177/ 1098612X14522052

8. Stieger K, Palos H, Giger U. Comparison of various blood-typing methods for the feline AB blood group system. Am J Vet Res. (2005) 66:13939. doi: 10.2460/ajvr.2005.66.1393

\section{ETHICS STATEMENT}

Ethical review and approval for this animal study was not required according to our institutional legislation because only leftover blood samples sent for routine diagnostic work were used. Written informed consent for further use of surplus material was obtained from the owner of the animals for the samples used in this study.

\section{AUTHOR'S NOTE}

This research was presented in part as poster at 60th American Association Veterinary Laboratory Diagnosticians (AAVLD)/121st United States Animal Health Association (USAHA) Annual Meeting, San Diego, CA, USA, 12-18 October 2017.

\section{AUTHOR CONTRIBUTIONS}

ES, RP, and DP contributed conception and design of the study; LB performed the laboratory analysis and organized the database; ES performed the statistical analysis, wrote the first draft of the manuscript. All authors contributed to manuscript revision, read, and approved the submission version.

\section{FUNDING}

This study was supported, in part, by Finanziamento Individuale per le Attività Base di Ricerca (FFABR), 2017.

9. Seth M, Jackson KV, Giger U. Comparison of five blood-typing methods for the feline AB blood group system. Am J Vet Res. (2011) 72:2039. doi: 10.2460/ajvr.72.2.203

10. Spada E, Proverbio D, Priolo V, Ippolito D, Baggiani L, Perego R, et al. Dog erythrocyte antigens (DEA) 1, 4, 7 and suspected naturally occurring anti-DEA 7 antibodies in Italian Corso dogs. Vet J. (2017) 222:1721. doi: 10.1016/j.tvjl.2017.02.008

11. Blais MC, Berman L, Oakley D, Giger U. Canine Dal blood type: A red cell antigen lacking in some Dalmatians. J Vet Intern Med. (2007) 21:2816. doi: 10.1111/j.1939-1676.2007.tb02961.x

12. Euler CC, Lee JH, Kim HY, Raj K, Mizukami R, Giger U. Survey of two new (Kai 1 and Kai 2) and other blood groups in dogs of North America. J Vet Intern Med. (2016) 30:1642-7. doi: 10.1111/jvim.14572

13. Butler M, Andrews GA, Smith JE. Reactivity of lectins with feline erythrocytes. Comp Haematol Int. (1991) 1:217-9. doi: 10.1007/ BF00235374

14. NCCLS. Method Comparison and Bias Estimation Using Patient Samples. Approved Guideline - Second Edition. NCCLS Document EP9-A2. NCCLS, Wayne, PA (2002).

15. Fleiss JL, Levin B, Paik MC. Statistical Methods for Rates and Proportions. 3rd edn. Hoboken, NY: John Wiley \& Sons (2003).

16. Altman DG. Practical Statistics for Medical Research. London: Chapman and Hall (1991).

17. Auer L, Bell K. Transfusion reactions in cats due to $A B$ blood group incompatibility. Res Vet Sci. (1983) 35:145-52. doi: 10.1016/ S0034-5288(18)32171-4

18. Giger U, Bucheler J. Transfusion of type-A and type-B blood to cats. J Am Vet Med Assoc. (1991) 198:411-8. 
19. Proverbio D, Spada E, Baggiani L, Perego R. Assessment of a gel column technique for feline blood typing. Vet Res Commun. (2009) 33:2013. doi: 10.1007/s11259-009-9275-7

20. Cooling L. ABO H. Lewis Blood Groups and Structurally Related Antigens, 18th edn. In: Fung MK, Grossman BJ, Hillyer CD, Westhoff CM, editors. AABB Technical manual. Bethesda, MD: AABB (2014). p. 219-315.

21. Walker PS, Harmening DM. Other technologies and Automation. In: Harmening DM, editor. Modern Blood Banking \& Transfusion Practices. Philadelphia, PA: F.A. Davis Company (2012). p. $273-87$.

22. Harmening DM, Forneris G, Tubby BJ. The ABO blood group system. In: Harmening DM, editor. Modern Blood Banking \& Transfusion Practices. Philadelphia, PA: F.A. Davis Company (2012). p. 119-48.

23. Abrams-Ogg ACG. Feline Recipient Screening. In: Yagi K, Holowaychuk MK, editors. Manual of Veterinary Transfusion Medicine and Blood Banking. Ames, IA: John Wiley \& Sons (2016). p. 129-54.

24. Weinstein NM, Blais M-C, Harris K, Oakley DA, Aronson LR, Giger U. A newly recognized blood group in domestic shorthair cats: the Mik red cell antigen. J Vet Intern Med. (2007) 21:287-92. doi: 10.1111 /j.1939-1676.2007.tb02962.x

25. Goy-Thollot I, Nectoux A, Guidetti M, Chaprier B, Bourgeois S, Boisvineau $\mathrm{C}$, et al. Detection of naturally occurring alloantibody by an in-clinic antiglobulin-enhanced and standard crossmatch gel column test in non- transfused domestic shorthair cats. J Vet Intern Med. (2019) 33:58895. doi: 10.1111/jvim.15381

26. McClosky ME, Cimino Brown D, Weinstein NM, Chappini N, Taney MT, Marryott K, et al. Prevalence of naturally occurring non-AB blood type incompatibilities in cats and influence of crossmatch on transfusion outcomes. J Vet Intern Med. (2018) 32:1934-42. doi: 10.1111/jvim.15334

27. Rizzi TE, Clinkenbeard KD, Meinkoth JH. Normal hematology of the cat, 6th edn. In: Weiss DJ, Wardrop JK, editors. Schalm's Veterinary Hematology. Ames, IA: Wiley-Blackwell (2010). p. 811-20.

28. Langston MM, Procter JL, Cipolone KM, Stroncek DF. Evaluation of the gel system for ABO groupig and D typing. Transfusion. (1999) 39:3005. doi: 10.1046/j.1537-2995.1999.39399219288.x

Conflict of Interest: The authors declare that the research was conducted in the absence of any commercial or financial relationships that could be construed as a potential conflict of interest.

Copyright (c) 2020 Spada, Perego, Baggiani and Proverbio. This is an open-access article distributed under the terms of the Creative Commons Attribution License (CC $B Y)$. The use, distribution or reproduction in other forums is permitted, provided the original author(s) and the copyright owner(s) are credited and that the original publication in this journal is cited, in accordance with accepted academic practice. No use, distribution or reproduction is permitted which does not comply with these terms. 